\title{
On the formal degree conjecture for simple supercuspidal representations
}

\author{
Yoichi Mieda
}

\begin{abstract}
We prove the formal degree conjecture for simple supercuspidal representations of symplectic groups and quasisplit even special orthogonal groups over a $p$-adic field, under the assumption that $p$ is odd. The essential part is to compute the Swan conductor of the exterior square of an irreducible local Galois representation with Swan conductor 1. It is carried out by passing to the equal characteristic local field and using the theory of Kloosterman sheaves.
\end{abstract}

\section{Introduction}

Let $F$ be a $p$-adic field, and $G$ a connected reductive group over $F$. For an irreducible discrete series representation $\pi$ of $G(F)$, we can consider an invariant $\operatorname{deg}(\pi) \in \mathbb{R}_{>0}$ called the formal degree of $\pi$. It is in some sense a generalization of the dimension (or the degree) of a finite-dimensional representation. On the other hand, by the local Langlands correspondence, irreducible smooth representations of $G(F)$ are conjecturally parametrized by pairs $(\phi, \rho)$, where $\phi: W_{F} \times \mathrm{SL}_{2}(\mathbb{C}) \rightarrow{ }^{L} G$ is an $L$ parameter, and $\rho$ is an irreducible representation of a finite group $\mathcal{S}_{\phi}$ determined by $\phi$. The formal degree conjecture, which was proposed by Hiraga-Ichino-Ikeda [HII08], predicts that $\operatorname{deg}(\pi)$ can be described by using the pair $\left(\phi_{\pi}, \rho_{\pi}\right)$ attached to $\pi$. For more precise formulation, see Section 3. This conjecture has been solved for general linear groups [HII08, odd special orthogonal groups [ILM17] and unitary groups $[\mathrm{Beu}]$, but it seems still open for many other groups.

In this article, we will focus on a very special class of discrete series representations, simple supercuspidal representations. They are introduced in GR10] and [RY14], and characterized among irreducible smooth representations by the property that they have minimal positive depth. The local Langlands correspondence for simple supercuspidal representations of quasi-split classical groups has been investigated in Oi's work [Oi] very precisely. As an application of his results, he obtained the following theorem:

Graduate School of Mathematical Sciences, The University of Tokyo, 3-8-1 Komaba, Meguro-ku, Tokyo, 153-8914, Japan

E-mail address: mieda@ms.u-tokyo.ac.jp

2010 Mathematics Subject Classification. Primary: 11F70; Secondary: 22E50. 
Yoichi Mieda

Theorem 1.1 ([Oi, Theorem 9.3]) Let $n \geq 1$ be an integer and write $2 n=p^{e} n^{\prime}$ with $p \nmid n^{\prime}$. Assume $p \neq 2$ and either $p \nmid 2 n$ or $n^{\prime} \mid p-1$. Let $G$ be one of the following groups:

$-\mathrm{Sp}_{2 n}$

- the quasi-split $\mathrm{SO}_{2 n}$ attached to a ramified quadratic extension of $F$,

- the split $\mathrm{SO}_{2 n+2}$,

- or the quasi-split $\mathrm{SO}_{2 n+2}$ attached to an unramified quadratic extension of $F$.

Then, the formal degree conjecture holds for simple supercuspidal representations of $G(F)$.

The goal of this article is to remove the condition "either $p \nmid 2 n$ or $n^{\prime} \mid p-1$ " in the theorem above. Here is our main theorem:

Theorem 1.2 (Theorem 3.5 and Remark 3.7) Assume $p \neq 2$. Let $G$ be one of the groups in Theorem 1.1. Then, the formal degree conjecture holds for simple supercuspidal representations of $G(F)$.

By the same method as in [Oi], Theorem 1.2 is easily reduced to the following:

Theorem 1.3 (Theorem 2.1) Let $\tau$ be a $2 n$-dimensional irreducible smooth representation of $W_{F}$ such that $\operatorname{Sw} \tau=1$. Then we have $\operatorname{Sw}\left(\wedge^{2} \tau\right)=n-1$.

In the case $p \mid 2 n$ and $n^{\prime} \mid p-1$ (which is more difficult than the case $p \nmid 2 n$ ), Oi used an explicit description of $\tau$ in [IT] to obtain the theorem above. Our strategy to Theorem 1.3 is totally different. First we use Deligne's result [Del84] to reduce Theorem 1.3 to the case where $F$ is an equal characteristic local field. In the equal characteristic case, every irreducible smooth representation of $W_{F}$ with Swan conductor 1 is essentially obtained as the localization at $\infty \in \mathbb{P}^{1}$ of a Kloosterman sheaf Kl (see $\left[\mathrm{SGA} 4 \frac{1}{2}\right.$, Sommes. trig.] and [Kat88]). The Swan conductor of the localization at $\infty$ of $\wedge^{2} \mathrm{Kl}$ can be computed by using the Grothendieck-Ogg-Shafarevich formula and the Grothendieck-Lefschetz trace formula.

The outline of this paper is as follows. In Section 2, we will compute the Swan conductor of the exterior square of an irreducible smooth representation $\tau$ of $W_{F}$ with Swan conductor 1. Although in Theorem 1.3 we assumed that $\operatorname{dim} \tau$ is even (in fact only this case is needed to prove Theorem 1.2), we will also treat the case where $\operatorname{dim} \tau$ is odd. In Section [3, after recalling the formal degree conjecture, we deduce Theorem 1.2 from Theorem 1.3 .

Acknowledgment This work was supported by JSPS KAKENHI Grant Number $15 \mathrm{H} 03605$.

Notation Every representation is considered over $\mathbb{C}$, unless otherwise noted. 


\section{Exterior square of local Galois representations with Swan conductor 1}

Let $p$ be a prime number and $F$ a finite extension of $\mathbb{Q}_{p}$. We write $k$ for the residue field of $F$ and $q$ for the cardinality of $k$. We fix an algebraic closure $\bar{F}$ of $F$ and put $\Gamma_{F}=\operatorname{Gal}(\bar{F} / F)$. Let $W_{F}$ denote the Weil group of $F$, that is, the subgroup of $\Gamma_{F}$ consisting of elements which induce integer powers of the Frobenius automorphism on the residue field $\bar{k}$ of $\bar{F}$. It is a locally compact group containing the inertia group $I_{F}$ as an open subgroup.

Recall that $\Gamma_{F}$ is equipped with the upper numbering ramification filtration $\left\{\Gamma_{F}^{j}\right\}_{j \in \mathbb{R}_{\geq 0}}$, which is a descending filtration consisting of open normal subgroups of $\Gamma_{F}$. The subgroup $\Gamma_{F}^{0}$ equals the inertia group $I_{F}$, and $\Gamma_{F}^{0+}$ equals the wild inertia group $P_{F}$. Here $\Gamma_{F}^{j+}$ denotes the closure of $\bigcup_{j^{\prime}>j} \Gamma_{F}^{j^{\prime}}$, as usual.

Let $V$ be a finite-dimensional smooth representation of $W_{F}$. It is known that there exists a unique direct sum decomposition $V=\bigoplus_{j \in \mathbb{R}_{\geq 0}} V_{j}$ as a representation of $P_{F}$, called the break decomposition, such that

$-V_{0}=V^{P_{F}}$, and

$-V_{j}^{\Gamma_{F}^{j}}=0$ and $V_{j}^{\Gamma_{F}^{j+}}=V_{j}$ for each $j \in \mathbb{R}_{>0}$

(see [Kat88, Proposition 1.1, Lemma 1.4]). The numbers $j$ with $V_{j} \neq 0$ are called the breaks of $V$. The Swan conductor Sw $V$ of $V$ is defined by

$$
\operatorname{Sw} V=\sum_{j \in \mathbb{R}_{\geq 0}} j \operatorname{dim} V_{j}
$$

Note that $\operatorname{Sw} V$ depends only on the restriction of $V$ to $P_{F}$.

Let $n \geq 1$ be an integer. In this section, we prove the following result.

Theorem 2.1 Let $(\tau, V)$ be an $n$-dimensional irreducible smooth representation of $W_{F}$ such that $\mathrm{Sw} \tau=1$. Then we have

$$
\mathrm{Sw}\left(\wedge^{2} \tau\right)= \begin{cases}m-1 & \text { if } n=2 m \text { is even, } \\ m & \text { if } n=2 m+1 \text { is odd. }\end{cases}
$$

If $n=1$, this theorem is obvious. Therefore, we assume $n \geq 2$ in the following. First we notice a simple lemma.

Lemma 2.2 Let $(\tau, V)$ be as in Theorem 2.1. Then we have $V^{P_{F}}=0$. Moreover, $V$ has only one break $1 / n$ and $\left.V\right|_{I_{F}}$ is irreducible.

Proof. Since $P_{F}$ is a normal subgroup of $W_{F}, V^{P_{F}}$ is a $W_{F}$-subrepresentation of $V$. The condition Sw $V=1$ implies that $V^{P_{F}} \neq V$. Therefore we have $V^{P_{F}}=0$ by the irreducibility of $V$. By [Kat88, Lemma 1.11], $V$ has only one break $1 / n$ and $\left.V\right|_{I_{F}}$ is irreducible. 
Yoichi Mieda

To prove Theorem 2.1, we will pass to the equal characteristic case. Put $F^{\prime}=$ $k((T))$, which is an equal characteristic local field. By Deligne's result [Del84], we can prove the following:

Lemma 2.3 Let $(\tau, V)$ be as in Theorem 2.1. Then there exists an $n$-dimensional irreducible smooth representation $\left(\tau^{\prime}, V^{\prime}\right)$ of $W_{F^{\prime}}$ such that

$$
\operatorname{Sw} \tau^{\prime}=\operatorname{Sw} \tau=1, \quad \operatorname{Sw}\left(\wedge^{2} \tau^{\prime}\right)=\operatorname{Sw}\left(\wedge^{2} \tau\right) .
$$

Proof. By [Del84, §3.5], there exists an isomorphism $\Gamma_{F} / \Gamma_{F}^{1} \cong \Gamma_{F^{\prime}} / \Gamma_{F^{\prime}}^{1}$, which is canonical up to inner automorphisms. By construction, it preserves the upper numbering ramification filtrations of $\Gamma_{F}$ and $\Gamma_{F^{\prime}}$. Further, [Del84, Proposition 3.6.1] tells us that it induces an isomorphism $W_{F} / \Gamma_{F}^{1} \cong W_{F^{\prime}} / \Gamma_{F^{\prime}}^{1}$. By using this isomorphism, we can construct a functor

- from the category of finite-dimensional smooth representations of $W_{F}$ whose breaks are less than 1

- to the category of finite-dimensional smooth representations of $W_{F^{\prime}}$ whose breaks are less than 1 .

Clearly this functor maps irreducible representations to irreducible representations, commutes with exterior products, and preserves the Swan conductors.

By Lemma 2.2, $\tau$ has only one break $1 / n$, which is less than 1 . Therefore we can take $\left(\tau^{\prime}, V^{\prime}\right)$ as the image of $(\tau, V)$ under this functor.

Now we use the Kloosterman sheaves introduced in [SGA4 $\frac{1}{2}$, Sommes. trig.] and Kat88. Let us recall their construction briefly. Take a prime number $\ell \neq p$. We fix an isomorphism $\overline{\mathbb{Q}}_{\ell} \cong \mathbb{C}$ and identify them. Note that an irreducible finitedimensional continuous representation of $W_{F}$ over $\overline{\mathbb{Q}}_{\ell}$ is automatically smooth, hence can be identified with an irreducible smooth representation of $W_{F}$ over $\mathbb{C}$. Let $\mathbb{P}^{1}$ denote the projective line over $k$, and put $\mathbb{A}^{1}=\mathbb{P}^{1} \backslash\{\infty\}, \mathbb{G}_{m}=\mathbb{P}^{1} \backslash\{0, \infty\}$. We consider the diagram

$$
\mathbb{G}_{m} \stackrel{\text { mult }}{\longleftarrow} \mathbb{G}_{m}^{n} \stackrel{\text { add }}{\longrightarrow} \mathbb{A}^{1}
$$

where the maps mult and add are given by $\left(x_{1}, \ldots, x_{n}\right) \mapsto x_{1} \cdots x_{n}$ and $\left(x_{1}, \ldots, x_{n}\right) \mapsto$ $x_{1}+\cdots+x_{n}$, respectively. We fix a non-trivial additive character $\psi: k \rightarrow \mathbb{C}^{\times}$, and write $\mathcal{L}_{\psi}$ for the Artin-Schreier sheaf on $\mathbb{A}^{1}$ corresponding to $\psi$. For multiplicative characters $\chi_{1}, \ldots, \chi_{n}: k^{\times} \rightarrow \mathbb{C}^{\times}$, we can construct the Kummer sheaf $\mathcal{K}_{\chi_{1}}, \ldots, \mathcal{K}_{\chi_{n}}$ on $\mathbb{G}_{m}$. We put

$$
\operatorname{Kl}\left(\chi_{1}, \ldots, \chi_{n}\right)=R \operatorname{mult}_{!}\left(\left(\mathcal{K}_{\chi_{1}} \otimes \cdots \otimes \mathcal{K}_{\chi_{n}}\right) \otimes \operatorname{add}^{*} \mathcal{L}_{\psi}\right)[-n+1] .
$$

If $\chi_{1}=\cdots=\chi_{n}=1$, we simply write $\mathrm{Kl}_{n}$ for $\mathrm{Kl}\left(\chi_{1}, \ldots, \chi_{n}\right)$. It is known that $\mathrm{Kl}\left(\chi_{1}, \ldots, \chi_{n}\right)$ is a smooth sheaf on $\mathbb{G}_{m}$ of rank $n$. Further, it enjoys the following properties:

$-\operatorname{Kl}\left(\chi_{1}, \ldots, \chi_{n}\right)_{\overline{0}}$, which is a representation of $\Gamma_{\text {Frac } \widehat{\mathcal{O}}_{\mathbb{1} 1,0}}$, is tamely ramified. 
On the formal degree conjecture for simple supercuspidal representations

$-\mathrm{Kl}\left(\chi_{1}, \ldots, \chi_{n}\right)_{\bar{\infty}}$, which is a representation of $\Gamma_{\mathrm{Frac}} \widehat{\mathcal{O}}_{\mathbb{P}_{1}, \infty}$, is totally wildly ramified with Swan conductor 1 (in particular it is irreducible by Kat88, Lemma 1.11]).

Here $\widehat{\mathcal{O}}_{\mathbb{P}^{1}, x}$ denotes the completion of the local ring $\mathcal{O}_{\mathbb{P}^{1}, x}$ at $x \in \mathbb{P}^{1}$. See $\operatorname{SGA} 4 \frac{1}{2}$, Sommes. trig., Théorème 7.8] and [Kat88, Theorem 4.1.1] for detail.

In the following, we fix an isomorphism $k[[T]] \cong \widehat{\mathcal{O}}_{\mathbb{P}^{1}, \infty}$ and identify them. Then $\mathrm{Kl}\left(\chi_{1}, \ldots, \chi_{n}\right)_{\infty}$ can be regarded as an $n$-dimensional irreducible smooth representation of $W_{F^{\prime}}$.

Lemma 2.4 Let $\tau^{\prime}$ be an $n$-dimensional irreducible smooth representation of $W_{F^{\prime}}$ with Swan conductor 1 . Then we have $\operatorname{Sw}\left(\wedge^{2} \tau^{\prime}\right)=\operatorname{Sw}\left(\wedge^{2} \mathrm{Kl}_{n, \bar{\infty}}\right)$.

Proof. By replacing $\tau^{\prime}$ by its unramified twist, we may assume that $\tau^{\prime}$ extends to a smooth representation of $\Gamma_{F^{\prime}}$. Note that $\tau^{\prime}$ is defined over a finite extension $E_{\lambda}$ of $\mathbb{Q}_{\ell}$ contained in $\overline{\mathbb{Q}}_{\ell}$. By the theorem of Katz-Gabber ([Kat86, Theorem 1.5.6]), there exists a smooth $E_{\lambda}$-sheaf $\mathcal{F}$ on $\mathbb{G}_{m}$ of rank $n$ such that $\mathcal{F}_{\overline{0}}$ is tamely ramified and $\mathcal{F}_{\bar{\infty}}$ is isomorphic to $\tau^{\prime}$. By [Kat88, Theorem 8.7.1] (see also the proof of [Kat88, Corollary 8.7.2]), there exist a finite extension $k^{\prime}$ of $k$, an element $a^{\prime} \in k^{\prime \times}$ and multiplicative characters $\chi_{1}^{\prime}, \ldots, \chi_{n}^{\prime}: k^{\prime \times} \rightarrow \mathbb{C}^{\times}$such that

$$
\mathcal{F} \otimes_{k} k^{\prime} \cong \iota_{a^{\prime}}^{*} \mathrm{Kl}\left(\chi_{1}^{\prime}, \ldots, \chi_{n}^{\prime}\right),
$$

where $\iota_{a^{\prime}}: \mathbb{G}_{m} \otimes_{k} k^{\prime} \rightarrow \mathbb{G}_{m} \otimes_{k} k^{\prime}$ is the multiplication by $a^{\prime}$ and $\operatorname{Kl}\left(\chi_{1}^{\prime}, \ldots, \chi_{n}^{\prime}\right)$ is the Kloosterman sheaf over $\mathbb{G}_{m} \otimes_{k} k^{\prime}$ with respect to the additive character $\psi \circ \operatorname{tr}_{k^{\prime} / k}$ of $k^{\prime}$. Since the base change from $k$ to $k^{\prime}$ and the pull-back by $\iota_{a^{\prime}}$ do not affect the Swan conductor at $\infty$, we conclude that $\operatorname{Sw}\left(\wedge^{2} \tau^{\prime}\right)=\operatorname{Sw}\left(\wedge^{2} \operatorname{Kl}\left(\chi_{1}^{\prime}, \ldots, \chi_{n}^{\prime}\right){ }_{\infty}\right)$. On the other hand, by [Kat88, Proposition 10.1], the restriction of $\operatorname{Kl}\left(\chi_{1}^{\prime}, \ldots, \chi_{n}^{\prime}\right)_{\infty}$ to $P_{k^{\prime}((T))}=P_{F^{\prime}}$ is independent of $\chi_{1}^{\prime}, \ldots, \chi_{n}^{\prime}$. Hence we have

$$
\begin{aligned}
\mathrm{Sw}\left(\wedge^{2} \mathrm{Kl}\left(\chi_{1}^{\prime}, \ldots, \chi_{n}^{\prime}\right)_{\bar{\infty}}\right) & =\mathrm{Sw}\left(\wedge^{2} \mathrm{Kl}\left(1^{\prime}, \ldots, 1^{\prime}\right)_{\bar{\infty}}\right)=\mathrm{Sw}\left(\wedge^{2}\left(\mathrm{Kl}_{n} \otimes_{k} k^{\prime}\right)_{\bar{\infty}}\right) \\
& =\mathrm{Sw}\left(\wedge^{2} \mathrm{Kl}_{n, \bar{\infty}}\right),
\end{aligned}
$$

where $1^{\prime}$ denotes the trivial character of $k^{\prime \times}$. This concludes the proof.

By Lemma 2.4, we may focus on computing $\operatorname{Sw}\left(\wedge^{2} \mathrm{Kl}_{n, \bar{\infty}}\right)$. Since $\wedge^{2} \mathrm{Kl}_{n, \overline{0}}$ is tame, the Grothendieck-Ogg-Shafarevich formula [SGA5, Exposé X, Théorème 7.1] tells us that

$$
\mathrm{Sw}\left(\wedge^{2} \mathrm{Kl}_{n, \bar{\infty}}\right)=-\chi_{c}\left(\mathbb{G}_{m}, \wedge^{2} \mathrm{Kl}_{n}\right):=-\sum_{i=0}^{2}(-1)^{i} \operatorname{dim} H_{c}^{i}\left(\mathbb{G}_{m} \otimes_{k} \bar{k}, \wedge^{2} \mathrm{Kl}_{n}\right) .
$$

We shall determine the Euler characteristic $\chi_{c}\left(\mathbb{G}_{m}, \wedge^{2} \mathrm{Kl}_{n}\right)$ by computing the $L$ function

$$
L\left(\mathbb{G}_{m}, \wedge^{2} \mathrm{Kl}_{n}, X\right)=\exp \left(\sum_{r=1}^{\infty}\left(\sum_{a \in k_{r}^{\times}} \operatorname{Tr}\left(\operatorname{Frob}_{a}, \wedge^{2} \mathrm{Kl}_{n, \bar{a}}\right)\right) \frac{X^{r}}{r}\right),
$$

where $k_{r}=\mathbb{F}_{q^{r}}$ denotes the degree $r$ extension of $k=\mathbb{F}_{q}$. 
Yoichi Mieda

Proposition 2.5 We have

$L\left(\mathbb{G}_{m}, \wedge^{2} \mathrm{Kl}_{n}, X\right)= \begin{cases}\frac{(1-q X)\left(1-q^{3} X\right) \cdots\left(1-q^{2 m-1} X\right)}{1-q^{2 m} X} & \text { if } n=2 m \text { is even, } \\ (1-q X)\left(1-q^{3} X\right) \cdots\left(1-q^{2 m-1} X\right) & \text { if } n=2 m+1 \text { is odd. }\end{cases}$

Proof. First note that

$$
\sum_{a \in k_{r}^{\times}} \operatorname{Tr}\left(\operatorname{Frob}_{a}, \wedge^{2} \mathrm{Kl}_{n, \bar{a}}\right)=\frac{1}{2}\left(\sum_{a \in k_{r}^{\times}} \operatorname{Tr}\left(\operatorname{Frob}_{a}, \mathrm{Kl}_{n, \bar{a}} \otimes \mathrm{Kl}_{n, \bar{a}}\right)-\sum_{a \in k_{r}^{\times}} \operatorname{Tr}\left(\operatorname{Frob}_{a}^{2}, \mathrm{Kl}_{n, \bar{a}}\right)\right) .
$$

By the proof of [Kat88, Proposition 10.4.1], we have

$$
\begin{aligned}
& \sum_{a \in k_{r}^{\times}} \operatorname{Tr}\left(\operatorname{Frob}_{a}, \mathrm{Kl}_{n, \bar{a}} \otimes \mathrm{Kl}_{n, \bar{a}}\right)=S_{r}(n, 1,1) \\
& \quad= \begin{cases}-1-q^{r}-q^{2 r}-\cdots-q^{(n-1) r}+q^{n r} & \text { if } n \text { is even or } p=2, \\
-1-q^{r}-q^{2 r}-\cdots-q^{(n-1) r} & \text { if } n \text { is odd and } p \neq 2\end{cases}
\end{aligned}
$$

(note that "if $\alpha(-\alpha)^{n} \beta=1$ " in the end of p. 173 of [Kat88] should be "if $\alpha(-\alpha)^{n} \beta=$ $-1 ")$. On the other hand, by [Kat88, (4.2.1.3), (4.2.1.5)], we have

$$
\sum_{a \in k_{r}^{\times}} \operatorname{Tr}\left(\operatorname{Frob}_{a}^{2}, \mathrm{Kl}_{n, \bar{a}}\right)=(-1)^{n-1} \frac{1}{q^{2 r}-1} \sum_{\rho \in\left(k_{2 r}^{\times}\right) \vee} g\left(\psi \circ \operatorname{tr}_{k_{2 r} / k}, \rho^{q^{r}-1}\right)^{n},
$$

where $\left(k_{2 r}^{\times}\right)^{\vee}$ denotes the set of characters of $k_{2 r}^{\times}$and

$$
g\left(\psi \circ \operatorname{tr}_{k_{2 r} / k}, \rho^{q^{r}-1}\right)=\sum_{a \in k_{2 r}^{\times}} \psi\left(\operatorname{tr}_{k_{2 r} / k}(a)\right) \rho(a)^{q^{r}-1}
$$

denotes the Gauss sum. Further, by [Kat88, (4.2.1.13)], we have

$$
g\left(\psi \circ \operatorname{tr}_{k_{2 r} / k}, \rho^{q^{r}-1}\right)= \begin{cases}q^{r} \rho(-1) & \text { if } \rho^{q^{r}-1} \neq 1 \\ -1 & \text { if } \rho^{q^{r}-1}=1 .\end{cases}
$$

Since

$$
\begin{gathered}
\#\left\{\rho \in\left(k_{2 r}^{\times}\right)^{\vee} \mid \rho^{q^{r}-1}=1\right\}=q^{r}-1, \\
\#\left\{\rho \in\left(k_{2 r}^{\times}\right)^{\vee} \mid \rho^{q^{r}-1} \neq 1, \rho(-1)=1\right\}= \begin{cases}\frac{\left(q^{r}-1\right)^{2}}{2} & \text { if } p \neq 2, \\
q^{2 r}-q^{r} & \text { if } p=2,\end{cases} \\
\#\left\{\rho \in\left(k_{2 r}^{\times}\right)^{\vee} \mid \rho^{q^{r}-1} \neq 1, \rho(-1)=-1\right\}= \begin{cases}\frac{q^{2 r}-1}{2} & \text { if } p \neq 2, \\
0 & \text { if } p=2,\end{cases}
\end{gathered}
$$


On the formal degree conjecture for simple supercuspidal representations

we have

$$
\sum_{a \in k_{r}^{\times}} \operatorname{Tr}\left(\operatorname{Frob}_{a}^{2}, \mathrm{Kl}_{n, \bar{a}}\right)= \begin{cases}\frac{1}{q^{r}+1}\left(\frac{(-1)^{n-1} q^{n r}\left(q^{r}-1\right)}{2}-\frac{q^{n r}\left(q^{r}+1\right)}{2}-1\right) & \text { if } p \neq 2, \\ \frac{(-1)^{n-1} q^{(n+1) r}-1}{q^{r}+1} & \text { if } p=2 .\end{cases}
$$

Now we assume that $n=2 m$ is even. Then we have

$$
\sum_{a \in k_{r}^{\times}} \operatorname{Tr}\left(\operatorname{Frob}_{a}^{2}, \mathrm{Kl}_{n, \bar{a}}\right)=-\frac{q^{(n+1) r}+1}{q^{r}+1}=-1+q^{r}-q^{2 r}+\cdots-q^{2 m r},
$$

hence

$$
\begin{aligned}
\sum_{a \in k_{r}^{\times}} \operatorname{Tr} & \left(\operatorname{Frob}_{a}, \wedge^{2} \mathrm{Kl}_{n, \bar{a}}\right) \\
= & \frac{1}{2}\left(\left(-1-q^{r}-q^{2 r}-\cdots-q^{(2 m-1) r}+q^{2 m r}\right)\right. \\
\quad & \left.\quad-\left(-1+q^{r}-q^{2 r}+\cdots+q^{(2 m-1) r}-q^{2 m r}\right)\right) \\
& =-q^{r}-q^{3 r}-\cdots-q^{(2 m-1) r}+q^{2 m r} .
\end{aligned}
$$

Therefore we conclude that

$$
L\left(\mathbb{G}_{m}, \wedge^{2} \mathrm{Kl}_{n}, X\right)=\frac{(1-q X)\left(1-q^{3} X\right) \cdots\left(1-q^{2 m-1} X\right)}{1-q^{2 m} X} .
$$

Next we consider the case where $n=2 m+1$ is odd and $p \neq 2$. We have

$$
\sum_{a \in k_{r}^{\times}} \operatorname{Tr}\left(\operatorname{Frob}_{a}^{2}, \mathrm{Kl}_{n, \bar{a}}\right)=-\frac{q^{n r}+1}{q^{r}+1}=-1+q^{r}-q^{2 r}+\cdots-q^{2 m r},
$$

hence

$$
\begin{aligned}
\sum_{a \in k_{r}^{\times}} & \operatorname{Tr}\left(\operatorname{Frob}_{a}, \wedge^{2} \mathrm{Kl}_{n, \bar{a}}\right) \\
= & \frac{1}{2}\left(\left(-1-q^{r}-q^{2 r}-\cdots-q^{(2 m-1) r}-q^{2 m r}\right)\right. \\
& \left.\quad-\left(-1+q^{r}-q^{2 r}+\cdots+q^{(2 m-1) r}-q^{2 m r}\right)\right) \\
& =-q^{r}-q^{3 r}-\cdots-q^{(2 m-1) r} .
\end{aligned}
$$

Therefore we conclude that

$$
L\left(\mathbb{G}_{m}, \wedge^{2} \mathrm{Kl}_{n}, X\right)=(1-q X)\left(1-q^{3} X\right) \cdots\left(1-q^{2 m-1} X\right) .
$$


Finally we assume that $n=2 m+1$ is odd and $p=2$. Then we have

$$
\sum_{a \in k_{r}^{\times}} \operatorname{Tr}\left(\operatorname{Frob}_{a}^{2}, \mathrm{Kl}_{n, \bar{a}}\right)=\frac{q^{(n+1) r}-1}{q^{r}+1}=-1+q^{r}-q^{2 r}+\cdots+q^{(2 m+1) r},
$$

hence

$$
\begin{aligned}
\sum_{a \in k_{r}^{\times}} & \operatorname{Tr}\left(\operatorname{Frob}_{a}, \wedge^{2} \mathrm{Kl}_{n, \bar{a}}\right) \\
= & \frac{1}{2}\left(\left(-1-q^{r}-q^{2 r}-\cdots-q^{(2 m-1) r}-q^{2 m r}+q^{(2 m+1) r}\right)\right. \\
& \left.\quad-\left(-1+q^{r}-q^{2 r}+\cdots-q^{2 m r}+q^{(2 m+1) r}\right)\right) \\
& =-q^{r}-q^{3 r}-\cdots-q^{(2 m-1) r} .
\end{aligned}
$$

Therefore we conclude that

$$
L\left(\mathbb{G}_{m}, \wedge^{2} \mathrm{Kl}_{n}, X\right)=(1-q X)\left(1-q^{3} X\right) \cdots\left(1-q^{2 m-1} X\right) .
$$

By the Grothendieck-Lefschetz trace formula, we have

$$
L\left(\mathbb{G}_{m}, \wedge^{2} \mathrm{Kl}_{n}, X\right)=\prod_{i=0}^{2} \operatorname{det}\left(1-X \text { Frob; } H_{c}^{i}\left(\mathbb{G}_{m} \otimes_{k} \bar{k}, \wedge^{2} \mathrm{Kl}_{n}\right)\right)^{(-1)^{i+1}}
$$

In particular we have $\operatorname{deg} L\left(\mathbb{G}_{m}, \wedge^{2} \mathrm{Kl} l_{n}, X\right)=-\chi_{c}\left(\mathbb{G}_{m}, \wedge^{2} \mathrm{Kl}_{n}\right)$. Hence we obtain the following corollary:

Corollary 2.6 We have

$$
\mathrm{Sw}\left(\wedge^{2} \mathrm{Kl}_{n, \bar{\infty}}\right)=-\chi_{c}\left(\mathbb{G}_{m}, \wedge^{2} \mathrm{Kl}_{n}\right)= \begin{cases}m-1 & \text { if } n=2 m \text { is even, } \\ m & \text { if } n=2 m+1 \text { is odd. }\end{cases}
$$

Now Theorem 2.1 follows from Lemmas 2.3, 2.4 and Corollary 2.6.

\section{The formal degree conjecture for simple super- cuspidal representations}

In this section, we deduce the formal degree conjecture for simple supercuspidal representations of symplectic groups and quasi-split even special orthogonal groups from Theorem 2.1. Let us first recall the conjecture quickly in the case of symplectic groups. For more detail, see [HII08].

In the following, we put $G=\operatorname{Sp}_{2 n}$ for an integer $n \geq 2$. We fix a non-trivial additive character $\psi: F \rightarrow \mathbb{C}^{\times}$and a Haar measure on $G(F)$. Let $(\pi, V)$ be an irreducible discrete series representation of $G(F)$. We fix a $G(F)$-invariant inner 
On the formal degree conjecture for simple supercuspidal representations

product $():, V \times V \rightarrow \mathbb{C}$. Then, there exists a unique positive real number $\operatorname{deg}(\pi)$, the formal degree of $\pi$, satisfying

$$
\int_{G(F)}(\pi(g) v, w) \overline{\left(\pi(g) v^{\prime}, w^{\prime}\right)} d g=\operatorname{deg}(\pi)^{-1}\left(v, v^{\prime}\right) \overline{\left(w, w^{\prime}\right)}
$$

for every $v, w, v^{\prime}, w^{\prime} \in V$. It depends on the fixed measure on $G(F)$, but is independent of the inner product $($,$) . The formal degree conjecture predicts that \operatorname{deg}(\pi)$ can be described by using the local Langlands correspondence.

By the local Langlands correspondence due to Arthur [Art13, discrete series representations of $G(F)$ are parametrized by pairs $(\phi, \rho)$, where

- $\phi: W_{F} \times \mathrm{SL}_{2}(\mathbb{C}) \rightarrow \widehat{G}(\mathbb{C})=\mathrm{SO}_{2 n+1}(\mathbb{C})$ is an $L$-parameter such that the centralizer group $S_{\phi}=\operatorname{Cent}_{\widehat{G}(\mathbb{C})}(\operatorname{Im} \phi)$ is finite,

- and $\rho$ is an irreducible representation of $\pi_{0}\left(S_{\phi}\right)=S_{\phi}$.

The pair attached to a discrete series representation $\pi$ is denoted by $\left(\phi_{\pi}, \rho_{\pi}\right)$.

Here is the statement of the formal degree conjecture for $\mathrm{Sp}_{2 n}$ :

Conjecture 3.1 ([HII08, Conjecture 1.4]) For an irreducible discrete series representation $\pi$ of $G(F)$, we have

$$
\operatorname{deg}(\pi)=C \cdot \frac{\operatorname{dim} \rho_{\pi}}{\# S_{\phi_{\pi}}}\left|\gamma\left(0, \operatorname{Ad} \circ \phi_{\pi}, \psi\right)\right| .
$$

Here

- $C \in \mathbb{R}_{>0}$ is a constant which depends only on the Haar measure on $G(F)$ and the non-trivial additive character $\psi$ (we may take $C=1$ by constructing a Haar measure carefully from $\psi$ ),

- Ad $\circ \phi_{\pi}$ is the composite of

$$
W_{F} \times \mathrm{SL}_{2}(\mathbb{C}) \stackrel{\phi_{\pi}}{\longrightarrow} \widehat{G}(\mathbb{C}) \stackrel{\operatorname{Ad}}{\longrightarrow} \mathrm{GL}(\operatorname{Lie} \widehat{G}(\mathbb{C}))
$$

- and

$$
\gamma\left(s, \operatorname{Ad} \circ \phi_{\pi}, \psi\right)=\varepsilon\left(s, \operatorname{Ad} \circ \phi_{\pi}, \psi\right) \frac{L\left(1-s, \operatorname{Ad} \circ \phi_{\pi}\right)}{L\left(s, \operatorname{Ad} \circ \phi_{\pi}\right)}
$$

denotes the local $\gamma$-factor.

Remark 3.2 In the case $G=\mathrm{Sp}_{2 n}, S_{\phi_{\pi}}$ is known to be an elementary 2-group, hence $\operatorname{dim} \rho_{\pi}=1$.

The formal degree conjecture for a general connected reductive group is formulated similarly, but one needs slight modification if the group is not simply connected. See [HII08] for detail. The formal degree conjecture has been proved for general linear groups [HII08, Theorem 3.1], odd special orthogonal groups [ILM17], and unitary groups [Beu], but it seems still open for many other groups, such as symplectic groups and even special orthogonal groups. 
In this paper, we focus on a very special class of discrete series representations, called simple supercuspidal representations. They are constructed by using the compact induction from a compact open subgroup of $G(F)$. Here we choose a symplectic form given by the skew-symmetric $2 n \times 2 n$ matrix

$$
\left(\begin{array}{lllll} 
& & & & 1 \\
& & & -1 & \\
& & & . \cdot & \\
& 1 & & & \\
-1 & & & &
\end{array}\right)
$$

to define $G=\operatorname{Sp}_{2 n}$. We define a sequence of compact open subgroups $G(F) \supset$ $I \triangleright I^{+} \triangleright I^{++}$as follows:

$$
\begin{aligned}
I & =\left(\begin{array}{ccc}
\mathcal{O}_{F} & & \mathcal{O}_{F} \\
& \ddots & \\
\mathfrak{p}_{F} & & \mathcal{O}_{F}
\end{array}\right), \quad I^{+}=\left(\begin{array}{ccc}
1+\mathfrak{p}_{F} & & \mathcal{O}_{F} \\
& \ddots & \\
\mathfrak{p}_{F} & & 1+\mathfrak{p}_{F}
\end{array}\right), \\
I^{++} & =\left(\begin{array}{cccc}
1+\mathfrak{p}_{F} & \mathfrak{p}_{F} & & \mathcal{O}_{F} \\
& \ddots & \ddots & \\
& \mathfrak{p}_{F} & \ddots & \mathfrak{p}_{F} \\
\mathfrak{p}_{F}^{2} & & & 1+\mathfrak{p}_{F}
\end{array}\right) .
\end{aligned}
$$

Here $\mathcal{O}_{F}$ denotes the ring of integers of $F$, and $\mathfrak{p}_{F}$ the maximal ideal of $\mathcal{O}_{F}$. If we fix a uniformizer $\varpi$ of $\mathcal{O}_{F}$, then we have an isomorphism

$$
I^{+} / I^{++} \cong k^{n+1} ;\left(a_{i j}\right) \mapsto\left(a_{12} \bmod \mathfrak{p}_{F}, \ldots, a_{n, n+1} \bmod \mathfrak{p}_{F}, \varpi^{-1} a_{2 n, 1} \bmod \mathfrak{p}_{F}\right) .
$$

A character of $I^{+} / I^{++} \cong k^{n+1}$ is said to be affine generic if it is non-trivial on each factor of $k^{n+1}$. Let $\chi$ be a character of $\pm I^{+}$such that $\left.\chi\right|_{I^{++}}$is trivial and $\left.\chi\right|_{I^{+}}$induces an affine generic character of $I^{+} / I^{++}$. Then, the compact induction $\mathrm{c}-\operatorname{Ind}_{ \pm I^{+}}^{G(F)} \chi$ is known to be irreducible supercuspidal. Representations obtained in this way are called simple supercuspidal representations.

The parameter $\left(\phi_{\pi}, \rho_{\pi}\right)$ attached to a simple supercuspidal representation $\pi$ is investigated by $\mathrm{Oi}$ in detail.

Theorem 3.3 ([Oi, Corollary 5.13, Theorem 7.17]) Assume $p \neq 2$. Let $\iota$ denote the embedding $\widehat{G}(\mathbb{C})=\mathrm{SO}_{2 n+1}(\mathbb{C}) \hookrightarrow \mathrm{GL}_{2 n+1}(\mathbb{C})$. For a simple supercuspidal representation $\pi$ of $G(F)$, we have the following:

- $\iota \circ \phi_{\pi}=\tau \oplus \omega$, where $\tau$ is an irreducible $2 n$-dimensional irreducible representation of $W_{F}$ with Swan conductor 1 and $\omega$ is a quadratic character of $W_{F}$. Furthermore, $\tau$ is orthogonal, that is, there exists a $W_{F}$-invariant non-degenerate symmetric bilinear form $\tau \times \tau \rightarrow \mathbb{C}$.

$-\# S_{\phi_{\pi}}=2$. 
On the formal degree conjecture for simple supercuspidal representations

Strictly speaking, Oi, Theorem 7.17] claims that $\iota \circ \phi_{\pi}=\tau \oplus \operatorname{det} \circ \tau$, where $\tau$ is the Langlands parameter of a simple supercuspidal representation of $\operatorname{GL}_{n}(F)$. However, it is well-known that such $\tau$ is irreducible and has Swan conductor 1; see [BH14, §2] for example. Further, since $\tau$ is orthogonal (see [Oi, Corollary 5.13]), the character $\omega=\operatorname{det} \circ \tau$ is quadratic.

By using Theorem 3.3, Oi obtained a partial result on the formal degree conjecture.

Theorem 3.4 ([@i, Theorem 9.3]) We write $2 n=p^{e} n^{\prime}$ with $p \nmid n^{\prime}$. Assume $p \neq 2$ and either $p \nmid 2 n$ or $n^{\prime} \mid p-1$. Then, Conjecture 3.1 holds for simple supercuspidal representations of $G(F)$.

In the case $p \mid 2 n$ and $n^{\prime} \mid p-1$, Oi used an explicit description of $\tau$ in Theorem 3.3 due to Imai and Tsushima [IT], which is extremely complicated. It involves 4 field extensions $F^{\mathrm{ur}} \subset E \subset T \subset M \subset N$ of the maximal unramified extension $F^{\mathrm{ur}}$ of $F$. The extension $N / E$ is always Galois, but the extension $N / F^{u r}$ is not necessarily Galois. The condition $n^{\prime} \mid p-1$ ensures that $N / F^{\text {ur }}$ is a Galois extension, which makes computations much simpler.

The following is our main theorem for symplectic groups (for quasi-split even special orthogonal groups, see Remark 3.7).

Theorem 3.5 Assume $p \neq 2$. Then, Conjecture 3.1 holds for simple supercuspidal representations of $G(F)$.

In fact, Theorem 3.5 can be deduced from Theorem 2.1 exactly in the same way as Oi did in [Oi, §9.3]. We include some of his arguments for reader's convenience. In the following, we assume that $p \neq 2$ and let $\pi$ be a simple supercuspidal representation of $G(F)$. Let $\tau$ and $\omega$ be as in Theorem 3.3.

Lemma 3.6 (i) We have $L\left(s, \operatorname{Ad} \circ \phi_{\pi}\right)=1$.

(ii) We have $\operatorname{Ar}\left(\operatorname{Ad} \circ \phi_{\pi}\right)=2 n^{2}+2 n$, where Ar denotes the Artin conductor.

Proof. First of all, note that $\operatorname{Ad} \circ \phi_{\pi}=\wedge^{2}(\tau \oplus \omega)=\wedge^{2} \tau \oplus \tau \otimes \omega$.

(i) It suffices to show that $\left(\operatorname{Ad} \circ \phi_{\pi}\right)^{I_{F}}=0$. Since $p \neq 2$, the quadratic character $\omega$ is tamely ramified. Therefore $(\tau \otimes \omega)^{P_{F}}=\tau^{P_{F}} \otimes \omega=0$ by Lemma 2.2. Hence it suffices to prove that $\left(\wedge^{2} \tau\right)^{I_{F}}=0$.

By Lemma 2.2, $\left.\tau\right|_{I_{F}}$ is irreducible, hence $\operatorname{dim}\left(\tau \otimes \tau^{\vee}\right)^{I_{F}}=1$. Since $\tau$ is orthogonal by Theorem 3.3 , we have

$$
1=\operatorname{dim}\left(\tau \otimes \tau^{\vee}\right)^{I_{F}}=\operatorname{dim}(\tau \otimes \tau)^{I_{F}}=\operatorname{dim}\left(\operatorname{Sym}^{2} \tau\right)^{I_{F}} \oplus \operatorname{dim}\left(\wedge^{2} \tau\right)^{I_{F}}
$$

and $\left(\operatorname{Sym}^{2} \tau\right)^{I_{F}}=\left(\operatorname{Sym}^{2} \tau\right)_{I_{F}} \neq 0$. Therefore we obtain $\left(\wedge^{2} \tau\right)^{I_{F}}=0$, as desired.

(ii) Since $\omega$ is tame, we have

$$
\operatorname{Sw}\left(\operatorname{Ad} \circ \phi_{\pi}\right)=\operatorname{Sw}\left(\wedge^{2} \tau\right)+\operatorname{Sw}(\tau \otimes \omega)=\operatorname{Sw}\left(\wedge^{2} \tau\right)+\operatorname{Sw}(\tau)=n
$$


by Theorem 2.1, On the other hand, by the proof of (i), we have

$$
\operatorname{dim}\left(\operatorname{Ad} \circ \phi_{\pi}\right) /\left(\operatorname{Ad} \circ \phi_{\pi}\right)^{I_{F}}=\operatorname{dim}\left(\operatorname{Ad} \circ \phi_{\pi}\right)=\frac{2 n(2 n+1)}{2}=2 n^{2}+n .
$$

Therefore we conclude that

$$
\operatorname{Ar}\left(\operatorname{Ad} \circ \phi_{\pi}\right)=\operatorname{Sw}\left(\operatorname{Ad} \circ \phi_{\pi}\right)+\operatorname{dim}\left(\operatorname{Ad} \circ \phi_{\pi}\right) /\left(\operatorname{Ad} \circ \phi_{\pi}\right)^{I_{F}}=2 n^{2}+2 n .
$$

Proof of Theorem 3.5. Let $\mathbf{S t}$ denote the Steinberg representation of $G(F)$. We may choose $\psi$ so that the following equalities hold:

$$
\begin{aligned}
\frac{\operatorname{deg}(\pi)}{\operatorname{deg}(\mathbf{S t})} & =\frac{q^{n^{2}+n}}{2 \gamma\left(0, \operatorname{Ad} \circ \phi_{\mathbf{S t}}, \psi\right)}, \\
\left|\varepsilon\left(0, \operatorname{Ad} \circ \phi_{\pi}, \psi\right)\right| & =q^{\frac{1}{2} \operatorname{Ar}\left(\operatorname{Ad} \circ \phi_{\pi}\right)} .
\end{aligned}
$$

See [GR10, (72)] for the first equality, and [GR10, (10) and Proposition 2.3] for the second. Together with Lemma [3.6, we obtain $\left|\gamma\left(0, \operatorname{Ad} \circ \phi_{\pi}, \psi\right)\right|=q^{n^{2}+n}$ and

$$
\operatorname{deg}(\pi)=\left|\frac{\operatorname{deg}(\mathbf{S t}) \gamma\left(0, \operatorname{Ad} \circ \phi_{\pi}, \psi\right)}{2 \gamma\left(0, \operatorname{Ad} \circ \phi_{\mathbf{S t}}, \psi\right)}\right| .
$$

On the other hand, by [HII08, §3.3], the formal degree conjecture for $\mathbf{S t}$ is known:

$$
\operatorname{deg}(\mathbf{S t})=C\left|\gamma\left(0, \operatorname{Ad} \circ \phi_{\mathbf{S t}}, \psi\right)\right|
$$

Hence we have

$$
\operatorname{deg}(\pi)=C \cdot \frac{1}{2}\left|\gamma\left(0, \operatorname{Ad} \circ \phi_{\pi}, \psi\right)\right|=C \cdot \frac{\operatorname{dim} \rho_{\pi}}{\# S_{\phi_{\pi}}}\left|\gamma\left(0, \operatorname{Ad} \circ \phi_{\pi}, \psi\right)\right|,
$$

as desired (recall that $\operatorname{dim} \rho_{\pi}=1$ by Remark 3.2 and $\# S_{\phi_{\pi}}=2$ by Theorem 3.3 )

Remark 3.7 As remarked in [Oi, §9], by using the results in [GI14, we can deduce from Theorem 3.5 the formal degree conjecture for simple supercuspidal representations of quasi-split even special orthogonal groups, under the assumption $p \neq 2$.

\section{References}

[Art13] J. Arthur, The endoscopic classification of representations: Orthogonal and symplectic groups, American Mathematical Society Colloquium Publications, vol. 61, American Mathematical Society, Providence, RI, 2013.

[Beu R. Beuzart-Plessis, Plancherel formula for $\mathrm{GL}_{n}(F) \backslash \mathrm{GL}_{n}(E)$ and applications to the Ichino-Ikeda and formal degree conjectures for unitary groups, arXiv:1812.00047. 
On the formal degree conjecture for simple supercuspidal representations

[BH14] C. J. Bushnell and G. Henniart, Langlands parameters for epipelagic representations of $\mathrm{GL}_{n}$, Math. Ann. 358 (2014), no. 1-2, 433-463.

[Del84] P. Deligne, Les corps locaux de caractéristique p, limites de corps locaux de caractéristique 0, Representations of reductive groups over a local field, Travaux en Cours, Hermann, Paris, 1984, pp. 119-157.

[GI14] W. T. Gan and A. Ichino, Formal degrees and local theta correspondence, Invent. Math. 195 (2014), no. 3, 509-672.

[GR10] B. H. Gross and M. Reeder, Arithmetic invariants of discrete Langlands parameters, Duke Math. J. 154 (2010), no. 3, 431-508.

[HII08] K. Hiraga, A. Ichino, and T. Ikeda, Formal degrees and adjoint $\gamma$-factors, J. Amer. Math. Soc. 21 (2008), no. 1, 283-304.

[ILM17] A. Ichino, E. Lapid, and Z. Mao, On the formal degrees of square-integrable representations of odd special orthogonal and metaplectic groups, Duke Math. J. 166 (2017), no. 7, 1301-1348.

[IT] N. Imai and T. Tsushima, Local Galois representations of Swan conductor one, preprint, arXiv:1509.02960.

[Kat86] N. M. Katz, Local-to-global extensions of representations of fundamental groups, Ann. Inst. Fourier (Grenoble) 36 (1986), no. 4, 69-106.

[Kat88] Gauss sums, Kloosterman sums, and monodromy groups, Annals of Mathematics Studies, vol. 116, Princeton University Press, Princeton, NJ, 1988.

[Oi] M. Oi, Simple supercuspidal L-packets of quasi-split classical groups, https://www.math.kyoto-u.ac.jp/ masaooi/ssc_classic.pdf

[RY14] M. Reeder and J.-K. Yu, Epipelagic representations and invariant theory, J. Amer. Math. Soc. 27 (2014), no. 2, 437-477.

[SGA $\left.4 \frac{1}{2}\right]$ P. Deligne, Cohomologie étale, Lecture Notes in Mathematics, Vol. 569, Springer-Verlag, Berlin, 1977.

[SGA5] Cohomologie l-adique et fonctions L (SGA5), Lecture Notes in Mathematics, Vol. 589, Springer-Verlag, Berlin, 1977. 